\title{
Trayectorias profesionales de la diáspora mexicana calificada
}

\author{
Professional trajectories of the qualified Mexican \\ diaspora
}

\section{Trajetórias profissionais da diáspora Mexicana qualificada}

\author{
Mónica Chávez Elorza ${ }^{1}$ \\ Docente investigadora \\ Unidad Académica en Estudios del Desarrollo \\ Universidad Autónoma de Zacatecas \\ monick.elorza@gmail.com \\ Yolanda Alfaro ${ }^{2}$ \\ Investigadora posdoctoral \\ Instituto de Investigaciones Económicas \\ Universidad Nacional Autónoma de México \\ yalfaro@iiec.unam.mx
}

Recibido: 20-10-17

Aprobado: 02-11-17

2 Doctorado en Estudios del Desarrollo. 


\section{Resumen}

Este artículo busca reconstruir las trayectorias migratorias, académicas y laborales de posgraduados mexicanos que residen en el exterior y pertenecen al Sistema Nacional de Investigadores. Para contrastar las razones académicas y laborales de la migración, en el análisis se tienen en cuenta tres categorías: área de estudio, país de estudios doctorales y sexo. La metodología para reconstruir las trayectorias es mixta: con enfoque cuantitativo, puesto que la información procede de la "Encuesta para talentos mexicanos residentes en el exterior"; y con enfoque cualitativo, ya que se analiza el curriculum vitae de una submuestra de estos investigadores. Se halló que la migración por estudios se lleva a cabo en edades inferiores a los 30 años y las estancias posdoctorales superiores a esa edad; la vivencia del sistema educativo e investigativo del país de destino permite tener trayectorias académicas más continuas. Las migraciones no se pueden atribuir a una u otra razón exclusivamente.

Palabras clave: trayectorias profesionales; migración calificada; México; Sistema Nacional de Investigadores.

\section{Abstract}

This paper seeks to reconstruct migratory, academic and labor trajectories of Mexican postgraduates living abroad that belong to the Sistema Nacional de Investigadores (National System of Researchers). In order to contrast both academic and labor reasons of migration, three categories are taken into account in the analysis: study area, country of doctoral studies and sex. Methodology used for reconstructing trajectories is mixed: with a quantitative approach, since the information comes from the "Survey for Mexican talents residing abroad"; and, with a qualitative approach, since curriculum vitae of a subsample of these researchers is analyzed. Results show that study migration takes place at ages less than 30 years-old and postdoctoral stays above this age; experiencing the educational and research system in the country of destination allows for more continuous academic trajectories. Migrations cannot be attributed to just one or another reason exclusively.

Keywords: Professional trajectories; qualified migration; Mexico; National System of Researchers.

\section{Resumo}

Este artigo busca reconstruir as trajetórias migratórias, acadêmicas e profissionais dos pós-graduados mexicanos que residem no exterior e pertencem ao Sistema Nacional de Pesquisadores. Consideram-se as categorias de área de estudo, país de estudos de doutorado e sexo para comparar os motivos da migração (estudos e trabalho). A metodologia é mista: se reconstroem as trajetórias com uma abordagem quantitativa, a partir da informação derivada da enquete de talentos mexicanos residentes no exterior, mas também abordam-se qualitativamente os currículum vitae de um grupo dos pesquisadores. Verificou-se que a migração por motivos de estudo ocorre em idades menores de 30 anos e as estâncias pós-doutorais superam essa idades. A inserção no sistema educativo e de pesquisa do país de destino, permite que tenham trajetórias acadêmicas mais contínuas. Além disso, as migrações não podem ser atribuídas a uma ou a outra razão exclusivamente.

Palavras-chave: trajetórias; migração qualificada; México; Sistema Nacional de Pesquisadores. 


\section{Introducción}

En las últimas décadas, ha habido un aumento en la selectividad de la migración calificada internacional. De acuerdo con Delgado-Wise, Chávez y Ramírez (2016) la tasa anual de crecimiento de la migración en general, entre 1990 y 2010, de los migrantes en general fue de 1,8, y la tasa anual de aquellos migrantes que cuentan con educación terciaria fue de 2,7. La estimación para la diáspora mexicana indica que en el 2013 el grupo de migrantes que contaba con al menos un año de educación superior sobrepasaba 2,2 millones, y que tan solo en Estados Unidos había 160 mil posgraduados, de los cuales, alrededor de $14 \mathrm{mil}$ contaba con título de doctorado (Gaspar y Chávez, 2016). A su vez, Lozano-Ascencio y Gandini (2012) reafirman este patrón en el periodo 2000-2010 y estiman un incremento de mexicanos con licenciatura de casi $75 \%$ y sumaban los 530 mil. Este aumento en el volumen de mexicanos migrantes calificados que se dirigen a Estados Unidos despertó interés en los académicos y políticos. Si bien el tema forma parte del campo de los estudios de migración y desarrollo desde la década de 1990, al momento se pueden identificar diversas agendas políticas-académicas, sobre todo en lo que respecta a la planificación del desarrollo científico y tecnológico del país.

Los estudios más recientes hacen énfasis, por ejemplo, en el aumento de la participación de las mujeres en la migración México-Estados Unidos, así como la manera en que los profesionales altamente calificados se insertan en el mercado laboral estadunidense (Ramírez-García y Gandini, 2017) o bien, los determinantes salariales de mujeres mexicanas migrantes calificadas con estudios en áreas de ciencia, tecnología, ingeniería y matemáticas (CTIM) en comparación con sus pares inmigrantes y nativas (Ramírez-García y Gandini, 2017; Chávez y Gaspar, 2018).

Tuirán y Ávila (2013) fueron de los primeros investigadores que resaltaron las consecuencias negativas para el desarrollo científico tecnológico del país de la llamada fuga de cerebros. Siguiendo ese tenor, recientemente, Delgado-Wise y Chávez (2016) profundizan en la importancia que tiene incluir a la masa crítica de migrantes en los procesos de innovación y desarrollo en el país. No obstante, es importante señalar que dichos autores abordan el tema de la migración calificada desde la visión de política económica, planteando que este flujo de migración es parte de la reestructuración de los sistemas nacionales de innovación; cuya característica central es el intercambio desigual en la división internacional del trabajo científico, pues con las políticas de atracción de profesionales altamente calificados se busca abaratar costos, transferir riesgos y apropiarse de los bienes comunes intangibles (Delgado-Wise y Chávez, 2015). Lozano-Ascencio, Gandini y Ramírez-García (2015), por su parte, argumentan que si bien los posgraduados en áreas CTIM son laboralmente más demandados en Estados Unidos que en México, las remuneraciones de estos posgraduados en el mercado estadounidense son menores en comparación con otros inmigrantes.

Otros trabajos, relativamente recientes, ponen en discusión la dimensión de la pérdida de talentos, a través de la identificación del país donde se obtuvo el título de licenciatura y/o posgrado. Dado que, dependiendo de la fuente de financiamiento de estos estudios, se puede o no hablar de una transferencia o pérdida no solo de recursos humanos sino también inversiones en educación; se pone en tela de juicio si se les debe categorizar como migrantes o no (Calva-Sánchez y Carrión, 2014). Calva-Sánchez (2014) opta por explorar a escala individual las motivaciones de estos mexicanos calificados para emigrar, utilizando como metodología la entrevista semiestructurada a profesionistas con títulos obtenidos en México y la construcción de trayectorias de vida; identifica tres etapas: antes de la migración, situación inmediata a la migración y situación actual.

Gaspar (2015) pone en discusión dicha interpretación al reconstruir la variable país de estudios (con base en la propuesta de Batalova et al., 2008); considera edad de arribo, tiempo de estancia en el país, edad actual y nivel de escolaridad formal del inmigrante. En su investigación concluye que de 886 mil migrantes mexicanos calificados solo $23,7 \%$ tiene estudios solo en Estados Unidos, 25,3\% tiene estudios en México y en Estados Unidos y el restante $51 \%$ tiene estudios únicamente 
en México (Gaspar, 2015) de manera que sí es posible hablar de una transferencia o pérdida de masa crítica.

Estos estudios nos permiten, por una parte, observar la necesidad de plantearse, desde la esfera individual, las motivaciones y/o razones en las que surgen estos procesos migratorios y cómo estos se articulan con las estructuras científicas y tecnológicas imperantes en los países de destino. Aunque en la agenda política y académica se tiende a conceptualizar la diáspora como un grupo homogéneo y se la menciona reiterativamente como un potencial para el desarrollo científico del país, sigue existiendo cierto desconocimiento de las características académicas y científicas de este cuerpo de profesionales; en particular, desde la perspectiva de trayectorias personales, laborales o educativas.

Lo anterior amerita poner en discusión los supuestos de las causas y los efectos de la migración de profesionales altamente calificados, tratando de utilizar categorías de análisis diferentes a la fuga de cerebros (brain drain), circulación de cerebros (brain circulation) y ganancia de cerebros (brain gain). Para ello, en este artículo se plantea en primer lugar, la necesidad de situar este flujo migratorio sur-norte en un contexto de profundas transformaciones en la organización, orientación, generación y apropiación del trabajo científico y tecnológico desde los países del norte global, que además trastoca la naturaleza y significados del sistema global de ciencia y tecnología. En segundo lugar, se propone contextualizar la pérdida y/o potencial de este cuerpo de profesionales en el exterior, a partir de las características que lo conforman, puesto que en la agenda política y académica se tiende a mencionar la pérdida o un potencial que representa para el desarrollo científico del país.

De manera que el objetivo del presente artículo es reconstruir las trayectorias migratorias, académicas y laborales de los posgraduados mexicanos que residen en el exterior y son miembros del Sistema Nacional de Investigadores (SNI). El tratamiento de la información contempló dos niveles de análisis. En un primer nivel, se muestran las cifras grosso modo de las respuestas que los encuestados dieron a cada una de las preguntas en las secciones de la encuesta: a) datos sociodemográficos; b) trayectoria educativa; c) situación laboral; d) contexto migratorio: razones para emigrar y para permanecer en el país de destino; e) producción científica, tecnológica, empresarial, cultural y artística; f) vinculación o interacción científica y tecnológica con personas y/o instituciones en México; g) propuestas de política pública para el retorno o vinculación en México; y h) adjuntar su curriculum vitae.

En un segundo nivel, para llevar a cabo el análisis de trayectorias académicas y migratorias se selecciona el curriculum vitae tomando en consideración la razón de la emigración indirecta, cuando una de las razones emigratorias fueron los estudios; y directa, cuando se mencionaron simultáneamente tres razones relacionadas a la cuestión laboral: mayores ingresos, mejores condiciones laborales y mayores oportunidades de trabajo.

El artículo está organizado en tres secciones. En la primera se revisan los conceptos teóricos y metodológicos sobre trayectorias y se expone la manera en que se retoman en este texto. En la segunda, se expone de manera descriptiva una caracterización sobre quiénes son y dónde están los miembros del SNI que residen en el exterior. En la tercera sección, se avanzó en la identificación de factores que configuran los perfiles según las siguientes categorías: área de conocimiento, sexo y país de estudios doctorales, haciendo uso de los currículos de una submuestra de estos investigadores. Finalmente, a manera de conclusiones, se proponen algunos elementos que permiten visualizar un perfil de los miembros del SNI que residen en el exterior de acuerdo con las trayectorias migratorias, educativas y laborales analizadas.

\section{Consideraciones teóricas y metodológicas}

El término de trayectoria se refiere a las distintas etapas que viven los sujetos ya sea en el ámbito educativo, laboral o, en el caso específico de este artículo, respecto a su proyecto migratorio. En tal 
sentido, se recupera la noción de trayectoria como un instrumento metodológico para delinear los perfiles a partir de la experiencia emigratoria en relación con el proyecto profesional-laboral que la orienta, y elaborar tipologías que faciliten obtener información que permita reconstruir las trayectorias según sexo y campo de conocimiento.

Para este propósito se analizaron las características de los miembros del $\mathrm{SNI}^{3}$ que residen en el exterior. El grupo de estudio es posgraduados y las trayectorias se reconstruyen con una metodología mixta; la parte cuantitativa se basa en el análisis de las respuestas de la encuesta para talentos en el exterior (2014)4 , y el análisis cualitativo deriva de una muestra de los currículos de los miembros del SNI que respondió dicha encuesta. De manera que el concepto de trayectoria que se utilizó corresponde al enfoque longitudinal de corte cuantitativo propuesto por Elder (1985), puesto que se trata de un enfoque que tiene como propósito tratar de identificar a lo largo del tiempo los factores que definen la realización de diferentes trayectorias de vida.

Articular la trayectoria migratoria, académica y laboral permite identificar y describir las relaciones que se establecen entre ellas, así como perfilar ciertos patrones de diferencia y similitudes entre dichas categorías.

El concepto de trayectoria migratoria, académica y laboral, además se complementa con algunos aspectos claves de la propuesta teórica de la perspectiva de la interseccionalidad, que abre el análisis de las trayectorias al complejo entramado

3 El Sistema Nacional de Investigadores, creado en 1984, busca promover y fortalecer, por medio de evaluación de pares, las actividades científico-tecnológicas en el país. Distingue a los investigadores como nivel candidato I, II, III y emérito, tanto nacional como en el extranjero; dicha distinción va acompañada por un estímulo económico.

4 La información utilizada proviene, por una parte, de la "Encuesta para talentos mexicanos residentes en el exterior" llevada a cabo en 2014, cuyo universo potencial y respuestas consistieron en 833 (164) SNI en el exterior y 3.241 (2.372) exbecarios Conacyt; con una tasa de respuesta del $24 \%$ y $23 \%$, respectivamente. Por otra parte, de una submuestra de los currículos de miembros del SNI que residen en el exterior. de las dimensiones de raza/etnia/nacionalidad, clase y género (Anthias, 2006; Ezquerra, 2008). Específicamente es tomada como una herramienta analítica para incorporar en el análisis de las tres trayectorias el área del conocimiento de los estudios doctorales diferenciándose por sexo, ya que dichas categorías se presentan como una dimensión clave para entender las particularidades que conforman los perfiles de investigadores e investigadoras.

\section{Panorama general de los miembros del Sistema Nacional de Investigadores en el Exterior}

\subsection{Características generales}

En general, se trata de 164 investigadores, 124 varones y 40 mujeres (cuadro 1). La edad promedio del grupo osciló en 37,6 años 38,2 varones y 35,7 mujeres). Los destinos son más diversos que el de la migración mexicana no calificada; Estados Unidos y Canadá concentran $47 \%$ y Europa 40,2\% de estos investigadores; asimismo, se aprecian destinos remotos como Australia (2,4\%), Rusia y Nueva Zelanda ( $0,6 \%$ cada uno).

En promedio, estos investigadores han permanecido 5,7 años en estos destinos y $86,4 \%$ domina el idioma que se habla en el lugar. Relacionado con el tiempo que llevan residiendo en el exterior, se observa que $48,8 \%$ cuenta con visa de trabajo, mientras que el estatus migratorio de $22 \%$ es residente permanente, $8,5 \%$ es ciudadano y $5,5 \%$ está naturalizado.

En cuanto al estado civil, alrededor del $71,3 \%$ está casado o en unión libre, mientras que $24,2 \%$ está soltero; para los unidos, se observa que $65 \%$ de sus parejas son de México. El nivel educativo de las parejas es alto, dado que $70,7 \%$ de ellos o ellas poseen estudios de posgrado o posdoctorado.

Con respecto a la condición de actividad laboral se encuentran dos grandes grupos, por una parte, quienes que se encuentran ocupados $(47,5 \%)$, generalmente en el sector de servicios investigación $(54,6 \%)$ o de educación $(24,5 \%) y$, por otra 
parte, quienes se encuentran realizando una estancia posdoctoral $(48,1 \%)$-son escasos los que están en búsqueda de empleo (3,7\%)-. Vale la pena señalar que los porcentajes muestran menor logro en la estabilidad ocupacional para las mujeres, 53,8\% declaró estar en una estancia posdoctoral, solo $41 \%$ ya se encuentra ocupada en una actividad laboral estable, mientras que 49,6\% de los varones declaró tener una actividad laboral estable en el momento de la encuesta.

Las desigualdades salariales entre mujeres y varones siguen estando presentes en estos niveles de calificación; mientras que $33 \%$ de los varones obtiene 60 mil dólares americanos anuales o más, solo $11,1 \%$ de las mujeres llega a recibir esa cantidad por su actividad laboral. La siguiente categoría, de 50 a 59 mil dólares anuales es similar, ya que frente a $15,7 \%$ de varones solo $5,6 \%$ de las mujeres percibe esa cantidad. En los niveles más bajos de ingresos, las mujeres aparecen con mayor porcentaje, pues en el rango de 20 a 29 mil dólares anuales, las mujeres representan $13,9 \%$ y los varones $12,2 \%$; y en el nivel de 10 a 19 mil dólares anuales, 2,8\% frente a 0,9\% de los varones.

Asimismo, se observa una segregación de tipo horizontal para ellas con relación al área del conocimiento en los estudios doctorales: medicina y ciencias salud (22,5\% vs. $9,7 \%$ ) y para ellos, en el área de ingeniería ( $16,9 \%$ vs. $10 \%)$ y en el área de física, matemáticas y ciencias de la tierra (32,5\% vs. $20 \%$ ). Asimismo, vale la pena subrayar que las áreas de administración, negocios y finanzas $(0,6 \%)$, ciencias sociales y económicas $(2,4 \%)$ y humanidades $(3 \%)$ son las que tienen menor representatividad tanto en varones como en mujeres. Esto último puede estar estar relacionado con los incentivos y/o reglas para acceder y posteriormente permanecer en el SNI; esto es, está relacionado con las actividades de ciencia y tecnología (CyT).

A pesar de lo anterior, tanto mujeres (97,1\%) como varones $(94,2 \%)$ señalaron que tienen una alta correspondencia entre la formación académica y las actividades laborales que desempeñan. No obstante, las respuestas sobre el grado de satisfacción con el puesto que tienen permiten intuir que el ingreso y, posiblemente, el ascenso estén haciéndose presentes, pues 55,6\% de las investigadoras indicaron tener un alto grado de satisfacción en comparación con el $77,3 \%$ de los investigadores varones; y más aún, $41,7 \%$ de las investigadoras presenta un grado medio de satisfacción versus $18,5 \%$ en el caso de los varones.

\subsection{Razones de la migración y vínculos con la formación educativa}

En la encuesta, se les solicitó que proporcionaran las tres razones más importantes por las cuales emigraron de México y las tres más importantes por las cuales permanecen en el país de destino (los porcentajes no suman cien; cuadro 2). Se aprecian en las primeras tres razones diferencias entre mujeres y varones. Para el $60 \%$ de ellas importa que haya mayor desarrollo profesional, seguido de mejores condiciones laborales (50\%) y de mayores oportunidades de trabajo (47\%). En el caso de los varones, $62,1 \%$ señala un mayor desarrollo profesional, seguido por razones de estudios $(57,3 \%$ ) y mayores oportunidades de trabajo $(45,2 \%)$. Existe mayor consistencia entre mujeres y varones con respecto a las razones para permanecer en el país de destino, en general, $43,3 \%$ indica que es por un mayor desarrollo profesional, 33,5\% señala que son las mayores oportunidades de trabajo, y mayores ingresos $32,9 \%$.

La experiencia migratoria, sea educativa y/o laboral, ha permitido adquirir, desarrollar y acumular capacidades y de ello están conscientes investigadores investigadoras, puesto que $44,2 \%$ señala que estas capacidades no las habrían podido obtener en México, y 49,1\% indica que las habría podido obtener en el país, pero de manera parcial. Esto es un indicativo del potencial que se puede lograr obtener a través de experiencias migratorias, de corto o largo plazo en otros ambientes educativos, laborales y organizacionales. Por ello, se les plateó la posibilidad de aplicar estas capacidades en México. El 85\% de los investigadores señaló que es posible aplicarlas, y 13,5\% indicó que solo de manera parcial. 
Trayectorias profesionales de la diáspora mexicana calificada

\begin{tabular}{|c|c|c|c|c|c|c|}
\hline \multirow[b]{2}{*}{ Descriptor } & \multicolumn{3}{|c|}{ Personas } & \multicolumn{3}{|c|}{ Porcentajes Columna } \\
\hline & Total & Hombres & Mujeres & Total & Hombres & Mujeres \\
\hline Residencia & 164 & 124 & 40 & 100,0 & 100,0 & 100,0 \\
\hline EE.UU. y Canadá & 77 & 56 & 21 & 47,0 & 45,2 & 52,5 \\
\hline Europa & 66 & 50 & 16 & 40,2 & 40,3 & 40,0 \\
\hline ALyC & 9 & 8 & 1 & 5,5 & 6,5 & 2,5 \\
\hline Australia & 4 & 4 & 0 & 2,4 & 3,2 & - \\
\hline Asia & 6 & 4 & 2 & 3,7 & 3,2 & 5,0 \\
\hline Nueva Zelanda & 1 & 1 & 0 & 0,6 & 0,8 & - \\
\hline Rusia & 1 & 1 & 0 & 0,6 & 0,8 & - \\
\hline Años país actual (promedio) & 5,7 & 6,0 & 4,9 & & & \\
\hline Edad promedio & 37,6 & 38,2 & 35,7 & & & \\
\hline Domina el idioma del país en que vive & 162 & 122 & 40 & 100,0 & 100,0 & 100,0 \\
\hline No & 22 & 15 & 7 & 13,6 & 12,3 & 17,5 \\
\hline Sí & 140 & 107 & 33 & 86,4 & 87,7 & 82,5 \\
\hline Estatus migratorio & 164 & 124 & 40 & 100,0 & 100,0 & 100,0 \\
\hline Visa de trabajo & 80 & 60 & 20 & 48,8 & 48,4 & 50,0 \\
\hline Residente permanente & 36 & 26 & 10 & 22,0 & 21,0 & 25,0 \\
\hline Visa de estudiante & 15 & 12 & 3 & 9,1 & 9,7 & 7,5 \\
\hline Ciudadano & 14 & 12 & 2 & 8,5 & 9,7 & 5,0 \\
\hline Naturalizado & 9 & 9 & 0 & 5,5 & 7,3 & - \\
\hline Otro (Jl, H4, Intercambio) & 8 & 3 & 5 & 4,9 & 2,4 & 12,5 \\
\hline Visa de turista & 1 & 1 & 0 & 0,6 & 0,8 & - \\
\hline Visa de reunificación familiar & 1 & 1 & 0 & 0,6 & 0,8 & - \\
\hline Estado conyugal & 164 & 124 & 40 & 100,0 & 100,0 & 100,0 \\
\hline Casada(o) civil o religiosamente & 97 & 76 & 21 & 59,1 & 61,3 & 52,5 \\
\hline Soltera(o) & 40 & 30 & 10 & 24,4 & 24,2 & 25,0 \\
\hline Unión libre & 20 & 12 & 8 & 12,2 & 9,7 & 20,0 \\
\hline Separada(o) & 3 & 3 & 0 & 1,8 & 2,4 & - \\
\hline Divorciada(o) & 4 & 3 & 1 & 2,4 & 2,4 & 2,5 \\
\hline *Lugar nacimiento pareja & 116 & 88 & 28 & 100,0 & 100,0 & 100,0 \\
\hline México & 76 & 59 & 17 & 65,5 & 67,0 & 60,7 \\
\hline Europa & 24 & 17 & 7 & 20,7 & 19,3 & 25,0 \\
\hline EEUỦ y Canadá & 7 & 6 & 1 & 6,0 & 6,8 & 3,6 \\
\hline $\mathrm{ALyC}$ & 5 & 3 & 2 & 4,3 & 3,4 & 7,1 \\
\hline Australia & 2 & 2 & 0 & 1,7 & 2,3 & - \\
\hline Asia & 2 & 1 & 1 & 1,7 & 1,1 & 3,6 \\
\hline${ }^{*}$ Nivel de escolaridad pareja & 116 & 88 & 28 & 100,0 & 100,0 & 100,0 \\
\hline Licenciatura & 26 & 24 & 2 & 22,4 & 27,3 & 7,1 \\
\hline Doctorado & 22 & 20 & 2 & 19,0 & 22,7 & 7,1 \\
\hline Posdoctorado & 34 & 20 & 14 & 29,3 & 22,7 & 50,0 \\
\hline Maestría & 26 & 18 & 8 & 22,4 & 20,5 & 28,6 \\
\hline Preparatoria & 4 & 3 & 1 & 3,4 & 3,4 & 3,6 \\
\hline Especialidad & 3 & 3 & 0 & 2,6 & 3,4 & - \\
\hline Sin instrucción & 1 & 0 & 1 & 0,9 & - & 3,6 \\
\hline Condición de actividad & 162 & 123 & 39 & 100,0 & 100,0 & 100,0 \\
\hline Ocupado(a) & 77 & 61 & 16 & 47,5 & 49,6 & 41,0 \\
\hline Realizando un posdoctorado & 78 & 57 & 21 & 48,1 & 46,3 & 53,8 \\
\hline En búsqueda de empleo & 6 & 5 & 1 & 3,7 & 4,1 & 2,6 \\
\hline Estudiante & 1 & 0 & 1 & 0,6 & - & 2,6 \\
\hline **Área del conocimiento & 164 & 124 & 40 & 100,0 & 100,0 & 100,0 \\
\hline Biología y química & 52 & 37 & 15 & 31,7 & 29,8 & 37,5 \\
\hline Físico-matemáticas y ciencias de la tierra & 47 & 39 & 8 & 28,7 & 31,5 & 20,0 \\
\hline Ingenierías & 25 & 21 & 4 & 15,2 & 16,9 & 10,0 \\
\hline Medicina y ciencias de la salud & 21 & 12 & 9 & 12,8 & 9,7 & 22,5 \\
\hline Biotecnología y ciencias agropecuarias & 9 & 6 & 3 & 5,5 & 4,8 & 7,5 \\
\hline Humanidades & 5 & 4 & 1 & 3,0 & 3,2 & 2,5 \\
\hline
\end{tabular}




\begin{tabular}{|c|c|c|c|c|c|c|}
\hline Ciencias sociales y económicas & 4 & 4 & 0 & 2,4 & 3,2 & - \\
\hline Administración, negocios y finanzas & 1 & 1 & 0 & 0,6 & 0,8 & - \\
\hline $\begin{array}{l}{ }^{* *} \text { Ingresos anuales en dólares } \\
\text { estadounidenses }\end{array}$ & 151 & 115 & 36 & 100,0 & 100,0 & 100,0 \\
\hline Menos de 10,000 & 4 & 4 & 0 & 2,6 & 3,5 & - \\
\hline De 10,000 a 19,999 & 2 & 1 & 1 & 1,3 & 0,9 & 2,8 \\
\hline De 20,000 a 29,999 & 19 & 14 & 5 & 12,6 & 12,2 & 13,9 \\
\hline De 30,000 a 39,999 & 27 & 20 & 7 & 17,9 & 17,4 & 19,4 \\
\hline De 40,000 a 49,999 & 26 & 16 & 10 & 17,2 & 13,9 & 27,8 \\
\hline De 50,000 a 59,999 & 20 & 18 & 2 & 13,2 & 15,7 & 5,6 \\
\hline 60,000 y más & 42 & 38 & 4 & 27,8 & 33,0 & 11,1 \\
\hline No aplica & 11 & 4 & 7 & 7,3 & 3,5 & 19,4 \\
\hline $\begin{array}{l}\text { Correspondencia entre formación académi- } \\
\text { ca y actividades laborales que desempeña }\end{array}$ & 155 & 120 & 35 & 100,0 & 100,0 & 100,0 \\
\hline Alta correspondencia & 147 & 113 & 34 & 94,8 & 94,2 & 97,1 \\
\hline Media correspondencia & 5 & 4 & 1 & 3,2 & 3,3 & 2,9 \\
\hline Baja correspondencia & 1 & 1 & 0 & 0,6 & 0,8 & - \\
\hline Ninguna correspondencia & 1 & 1 & 0 & 0,6 & 0,8 & - \\
\hline No aplica & 1 & 1 & 0 & 0,6 & 0,8 & - \\
\hline $\begin{array}{l}\text { Grado de satisfacción con el puesto que } \\
\text { desempeña }\end{array}$ & 155 & 119 & 36 & 100,0 & 100,0 & 100,0 \\
\hline Alto & 112 & 92 & 20 & 72,3 & 77,3 & 55,6 \\
\hline Medio & 37 & 22 & 15 & 23,9 & 18,5 & 41,7 \\
\hline Bajo & 3 & 3 & 0 & 1,9 & 2,5 & - \\
\hline No aplica & 3 & 2 & 1 & 1,9 & 1,7 & 2,8 \\
\hline
\end{tabular}

Notas: *Aplica a los que respondieron casados civil o religiosamente y en unión libre. ** Área del conocimiento de doctorado. ${ }^{* * *}$ No aplica para los que buscan empleo.

Fuente: Encuesta para talentos mexicanos residentes en el exterior, Conacyt-UNESCO MOST México, 2014.

\begin{tabular}{|c|c|c|c|c|c|c|}
\hline \multirow[b]{2}{*}{ Descriptor } & \multicolumn{3}{|c|}{ Personas } & \multicolumn{3}{|c|}{ Porcentajes Columna } \\
\hline & Total & Hombres & Mujeres & Total & Hombres & Mujeres \\
\hline $\begin{array}{l}\text { Tres razones más importantes por las que } \\
\text { emigró }\end{array}$ & 164 & 124 & 40 & & & \\
\hline Mayor desarrollo profesional & 101 & 77 & 24 & 61,6 & 62,1 & 60,0 \\
\hline Estudios & 87 & 71 & 16 & 53,0 & 57,3 & 40,0 \\
\hline Mayores oportunidades de trabajo & 75 & 56 & 19 & 45,7 & 45,2 & 47,5 \\
\hline Mejores condiciones laborales & 65 & 45 & 20 & 39,6 & 36,3 & 50,0 \\
\hline Mayores ingresos & 39 & 26 & 13 & 23,8 & 21,0 & 32,5 \\
\hline Por la inseguridad en México & 37 & 25 & 12 & 22,6 & 20,2 & 30,0 \\
\hline Por motivos familiares & 13 & 8 & 5 & 7,9 & 6,5 & 12,5 \\
\hline Por compromiso laboral en la empresa & 1 & 1 & 0 & 0,6 & 0,8 & 0,0 \\
\hline Otro & 14 & 14 & 0 & 8,5 & 11,3 & 0,0 \\
\hline $\begin{array}{l}\text { Tres razones más importantes por las que } \\
\text { permanece en el exterior }\end{array}$ & 164 & 124 & 40 & & & \\
\hline Mayor desarrollo profesional & 71 & 57 & 14 & 43,3 & 46,0 & 35,0 \\
\hline Mayores oportunidades de trabajo & 55 & 44 & 11 & 33,5 & 35,5 & 27,5 \\
\hline Mayores ingresos & 54 & 44 & 10 & 32,9 & 35,5 & 25,0 \\
\hline Mejores condiciones laborales & 52 & 42 & 10 & 31,7 & 33,9 & 25,0 \\
\hline Por la inseguridad en México & 33 & 24 & 9 & 20,1 & 19,4 & 22,5 \\
\hline Por motivos familiares & 29 & 21 & 8 & 17,7 & 16,9 & 20,0 \\
\hline Estudios & 15 & 11 & 4 & 9,1 & 8,9 & 10,0 \\
\hline Por compromiso laboral en la empresa & 8 & 7 & 1 & 4,9 & 5,6 & 2,5 \\
\hline Otro & 10 & 9 & 1 & 6,1 & 7,3 & 2,5 \\
\hline
\end{tabular}


Trayectorias profesionales de la diáspora mexicana calificada

$\overline{\text { Cuadro 2. Razones para emigrar y vínculos con la formación educativa de los miembros del Sistema Nacional }}$ de Investigadores en el Exterior

\begin{tabular}{|c|c|c|c|c|c|c|}
\hline \multirow[b]{2}{*}{ Descriptor } & \multicolumn{3}{|c|}{ Personas } & \multicolumn{3}{|c|}{ Porcentajes Columna } \\
\hline & Total & Hombres & Mujeres & Total & Hombres & Mujeres \\
\hline Disposición para retornar a México & 160 & 122 & 38 & 100,0 & 100,0 & 100,0 \\
\hline No retornaría bajo ninguna circunstancia & 5 & 4 & 1 & 3,1 & 3,3 & 2,6 \\
\hline Retornaría de manera temporal & 41 & 31 & 10 & 25,6 & 25,4 & 26,3 \\
\hline Retornaría de manera permanente & 114 & 87 & 27 & 71,3 & 71,3 & 71,1 \\
\hline $\begin{array}{l}\text { Ha recibido algún reconocimiento en su } \\
\text { campo }\end{array}$ & 153 & 116 & 37 & 100,0 & 100,0 & 100,0 \\
\hline No & 51 & 36 & 15 & 33,3 & 31,0 & 40,5 \\
\hline Sí & 102 & 80 & 22 & 66,7 & 69,0 & 59,5 \\
\hline $\begin{array}{l}\text { ¿Considera que las capacidades adquiridas } \\
\text { en el extranjero las podría haber adquirido } \\
\text { en México? }\end{array}$ & 163 & 124 & 39 & 100,0 & 100,0 & 100,0 \\
\hline No & 72 & 54 & 18 & 44,2 & 43,5 & 46,2 \\
\hline Sí, parcialmente & 80 & 61 & 19 & 49,1 & 49,2 & 48,7 \\
\hline Sí & 11 & 9 & 2 & 6,7 & 7,3 & 5,1 \\
\hline $\begin{array}{l}\text { ¿Considera que las capacidades que } \\
\text { adquirió en el extranjero podrían aplicarse } \\
\text { para impulsar el desarrollo de México? }\end{array}$ & 163 & 124 & 39 & 100,0 & 100,0 & 100,0 \\
\hline No & 2 & 1 & 1 & 1,2 & 0,8 & 2,6 \\
\hline Sí, parcialmente & 22 & 17 & 5 & 13,5 & 13,7 & 12,8 \\
\hline Sí & 139 & 106 & 33 & 85,3 & 85,5 & 84,6 \\
\hline $\begin{array}{l}\text { En los últimos tres años ¿ha tenido alguna } \\
\text { vinculación/interacción con personas o } \\
\text { instituciones en México para generar } \\
\text { alguno de los siguientes productos? }\end{array}$ & 164 & 124 & 40 & & & \\
\hline Ninguno & 47 & 36 & 11 & 28,7 & 29,0 & 27,5 \\
\hline Artículos científicos & 90 & 70 & 20 & 54,9 & 56,5 & 50,0 \\
\hline Capítulos de libro & 28 & 24 & 4 & 17,1 & 19,4 & 10,0 \\
\hline Artículos de divulgación & 24 & 18 & 6 & 14,6 & 14,5 & 15,0 \\
\hline Libros & 13 & 12 & 1 & 7,9 & 9,7 & 2,5 \\
\hline Innovacion: producto & 7 & 6 & 1 & 4,3 & 4,8 & 2,5 \\
\hline Innovación: proceso & 5 & 4 & 1 & 3,0 & 3,2 & 2,5 \\
\hline Patentes & 4 & 4 & 0 & 2,4 & 3,2 & 0,0 \\
\hline Desarrollo de empresas & 3 & 3 & 0 & 1,8 & 2,4 & 0,0 \\
\hline Creación de empresas & 3 & 3 & 0 & 1,8 & 2,4 & 0,0 \\
\hline Innovación: organización o mercado & 0 & 0 & 0 & 0,0 & 0,0 & 0,0 \\
\hline Otro & 28 & 23 & 5 & 17,1 & 18,5 & 12,5 \\
\hline $\begin{array}{l}\text { De las siguientes opciones de política pública, } \\
\text { marque las tres que considere más importantes } \\
\text { para impulsar el retorno del talento mexicano } \\
\text { residente en el exterior }\end{array}$ & 164 & 124 & 40 & & & \\
\hline $\begin{array}{l}\text { * Promover una oferta laboral competitiva } \\
\text { internacionalmente }\end{array}$ & 144 & 108 & 36 & 87,8 & 87,1 & 90,0 \\
\hline $\begin{array}{l}\text { * Ampliar la infraestructura apropiada para el } \\
\text { desarrollo científico y tecnológico }\end{array}$ & 132 & 100 & 32 & 80,5 & 80,6 & 80,0 \\
\hline $\begin{array}{l}\text { * Promover la diversificación de esquemas de } \\
\text { financiamiento para la investigación, ágiles y } \\
\text { oportunos }\end{array}$ & 94 & 68 & 26 & 57,3 & 54,8 & 65,0 \\
\hline $\begin{array}{l}\text { * Impulsar una mayor vinculación entre la } \\
\text { academia y el aparato productivo }\end{array}$ & 52 & 35 & 17 & 31,7 & 28,2 & 42,5 \\
\hline $\begin{array}{l}\text { * Generar programas para el traslado y la } \\
\text { integración familiar }\end{array}$ & 34 & 26 & 8 & 20,7 & 21,0 & 20,0 \\
\hline $\begin{array}{l}\text { * Profesionalización de gestores para la promo- } \\
\text { ción de la producción científico y tecnológico }\end{array}$ & 22 & 16 & 6 & 13,4 & 12,9 & 15,0 \\
\hline
\end{tabular}
la vinculación con el aparato productivo 


\begin{tabular}{|c|c|c|c|c|c|c|}
\hline \multirow[b]{2}{*}{ Descriptor } & \multicolumn{3}{|c|}{ Personas } & \multicolumn{3}{|c|}{ Porcentajes Columna } \\
\hline & Total & Hombres & Mujeres & Total & Hombres & Mujeres \\
\hline $\begin{array}{l}\text { * Incrementar la promoción y difusión de la } \\
\text { producción artística y cultural }\end{array}$ & 16 & 13 & 3 & 9,8 & 10,5 & 7,5 \\
\hline $\begin{array}{l}\text { * Consolidar el sistema nacional de patentes (ágil, } \\
\text { accesible y competitivo internacionalmente) }\end{array}$ & 12 & 8 & 4 & 7,3 & 6,5 & 10,0 \\
\hline * Gestionar una mejor oferta de capital de riesgo & 11 & 10 & 1 & 6,7 & 8,1 & 2,5 \\
\hline $\begin{array}{l}\text { * Garantizar la seguridad y respeto a la propiedad } \\
\text { empresarial e intelectual }\end{array}$ & 11 & 7 & 4 & 6,7 & 5,6 & 10,0 \\
\hline $\begin{array}{l}\text { * Crear un portafolio de oportunidades de } \\
\text { inversión }\end{array}$ & 11 & 8 & 3 & 6,7 & 6,5 & 7,5 \\
\hline * Otro & 10 & 8 & 2 & 6,1 & 6,5 & 5,0 \\
\hline
\end{tabular}

Fuente: Encuesta para talentos mexicanos residentes en el exterior, Conacyt-UNESCO MOST México, 2014.

Adicionalmente, se les planteó su disposición para retornar a México, y en general, 71,3\% del total indicó que lo haría de manera permanente, $25,6 \%$ de manera temporal y solo un 3,1\% no retornaría bajo ninguna circunstancia. En la encuesta también se exploró sobre los tipos de vínculos de colaboración que mantienen de manera individual y/o institucional en México. Para ello se les preguntó si produjeron algún producto técnico, científico o innovativo como una forma de establecer un vínculo laboral o académico con México. Un cuarto del total de entrevistados no realizó ninguno de los productos mencionados. El 86,6\% indicó vinculación en la producción de artículos científicos de divulgación y/o de capítulos de libro. Solo $1,8 \%$ señaló alguna vinculación para la creación o desarrollo de empresas y $2,4 \%$ para producir patentes.

En este sentido, indicaron que para que una política de retorno resulte atractiva y eficaz para investigadores/as residentes en el extranjero debe considerar los siguientes aspectos:a) promover una oferta laboral competitiva internacionalmente $(87,8 \%)$; b) ampliar la infraestructura apropiada para el desarrollo científico y tecnológico $(80,5 \%)$; c) promover la diversificación de esquemas de financiamiento para la investigación, ágiles $y$ oportunos (57,3\%); d) impulsar una mayor vinculación entre la academia y el aparato productivo (31,7\%); y e) generar programas para el traslado y la integración familiar (31,7\%).

\subsection{Trayectorias académicas y el rol central del Conacyt en la formación de posgraduados}

Las trayectorias educativas que han seguido los investigadores deben analizarse tomando en consideración el rol que ha tenido el Consejo Nacional de Ciencia y Tecnología (Conacyt) a través de la oferta de becas para posgrado nacional e internacional, de la promoción y generación de programas formativos de calidad a través del Programa Nacional de Posgrado de Calidad (PNPC), del financiamiento para realizar estancias posdoctorales, tanto nacionales como internacionales y de la creación de las Cátedras para Jóvenes Investigadores (cuadro 3).

Dicho lo anterior, se tiene que $98,2 \%$ de entrevistados realizaron sus estudios de licenciatura o equivalente en México. Como es de esperarse, conforme avanza el grado académico es más común un aumento de investigadores/as realizando sus estudios en el exterior. Así entonces, de quienes cuentan con una especialidad alrededor de $67,6 \%$ la hizo en México, 14,7\% en Europa y $8,8 \%$ en Japón. En el caso de estudios de maestría, $68,9 \%$ la realizó en México, 17,4\% en Europa y $10,6 \%$ en Estados Unidos o Canadá. Para nivel de doctorado, $47,2 \%$ lo cursó en México; 32,5\% en Europa y 16,6\% en Estados Unidos o Canadá. En las estancias posdoctorales se observa que 5,3\% la hizo en México, 44\% en Estados Unidos o Canadá y 40,7\% en algún país europeo. 
Trayectorias profesionales de la diáspora mexicana calificada

Cuadro 3. Trayectorias académicas y el rol central del Conacyt en la formación educativa de los miembros del Sistema Nacional de Investigadores en el Exterior

\begin{tabular}{|c|c|c|c|c|c|c|}
\hline \multirow[b]{2}{*}{ Descriptor } & \multicolumn{3}{|c|}{ Personas } & \multicolumn{3}{|c|}{ Porcentajes Columna } \\
\hline & Total & Hombres & Mujeres & Total & Hombres & Mujeres \\
\hline Lugar de estudios Licenciatura & 164 & 124 & 40 & 100,0 & 100,0 & 100,0 \\
\hline México & 161 & 121 & 40 & 98,2 & 97,6 & 100,0 \\
\hline Europa & 2 & 2 & 0 & 1,2 & 1,6 & 0,0 \\
\hline Colombia & 1 & 1 & 0 & 0,6 & 0,8 & 0,0 \\
\hline Lugar de estudios Especialidad & 34 & 26 & 8 & 100,0 & 100,0 & 100,0 \\
\hline México & 23 & 15 & 8 & 67,6 & 57,7 & 100,0 \\
\hline Europa & 5 & 5 & 0 & 14,7 & 19,2 & 0,0 \\
\hline Japón & 3 & 3 & 0 & 8,8 & 11,5 & 0,0 \\
\hline Colombia & 1 & 1 & 0 & 2,9 & 3,8 & 0,0 \\
\hline Estados Unidos & 2 & 2 & 0 & 5,9 & 7,7 & 0,0 \\
\hline Lugar de estudios Maestría & 132 & 101 & 31 & 100,0 & 100,0 & 100,0 \\
\hline México & 91 & 66 & 25 & 68,9 & 65,3 & 80,6 \\
\hline Europa & 23 & 18 & 5 & 17,4 & 17,8 & 16,1 \\
\hline EEUU y Canadá & 14 & 13 & 1 & 10,6 & 12,9 & 3,2 \\
\hline Nueva Zelanda & 3 & 3 & 0 & 2,3 & 3,0 & 0,0 \\
\hline Japón & 1 & 1 & 0 & 0,8 & 1,0 & 0,0 \\
\hline Lugar de estudios Doctorado & 163 & 123 & 40 & 100,0 & 100,0 & 100,0 \\
\hline México & 77 & 55 & 22 & 47,2 & 44,7 & 55,0 \\
\hline Europa & 53 & 40 & 13 & 32,5 & 32,5 & 32,5 \\
\hline EEUỦ y Canadá & 27 & 22 & 5 & 16,6 & 17,9 & 12,5 \\
\hline Japón & 3 & 3 & 0 & 1,8 & 2,4 & 0,0 \\
\hline Nueva Zelanda & 3 & 3 & 0 & 1,8 & 2,4 & 0,0 \\
\hline Lugar de estancia postdoctoral & 150 & 112 & 38 & 100,0 & 100,0 & 100,0 \\
\hline Europa & 61 & 47 & 14 & 40,7 & 42,0 & 36,8 \\
\hline EEUỦ y Canadá & 66 & 46 & 20 & 44,0 & 41,1 & 52,6 \\
\hline Asia & 5 & 3 & 2 & 3,3 & 2,7 & 5,3 \\
\hline México & 8 & 7 & 1 & 5,3 & 6,3 & 2,6 \\
\hline Nueva Zelanda & 1 & 1 & 0 & 0,7 & 0,9 & 0,0 \\
\hline Australia & 4 & 4 & 0 & 2,7 & 3,6 & 0,0 \\
\hline Rusia & 1 & 1 & 0 & 0,7 & 0,9 & 0,0 \\
\hline ALyC & 4 & 3 & 1 & 2,7 & 2,7 & 2,6 \\
\hline Contó con beca en la licenciatura & 163 & 123 & 40 & 100,0 & 100,0 & 100,0 \\
\hline No & 96 & 70 & 26 & 58,9 & 56,9 & 65,0 \\
\hline Conacyt & 9 & 6 & 3 & 5,5 & 4,9 & 7,5 \\
\hline $\begin{array}{l}\text { Gobierno, sector privado, fundaciones } \\
\text { (mexicanas) }\end{array}$ & 46 & 36 & 10 & 28,2 & 29,3 & 25,0 \\
\hline Gobierno extranjero & 1 & 1 & 0 & 0,6 & 0,8 & 0,0 \\
\hline No aplica & 11 & 10 & 1 & 6,7 & 8,1 & 2,5 \\
\hline Contó con beca en la especialidad & 27 & 20 & 7 & 100,0 & 100,0 & 100,0 \\
\hline No & 7 & 4 & 3 & 25,9 & 20,0 & 42,9 \\
\hline Conacyt & 6 & 5 & 1 & 22,2 & 25,0 & 14,3 \\
\hline $\begin{array}{l}\text { Gobierno, sector privado, fundaciones } \\
\text { (mexicanas) }\end{array}$ & 4 & 2 & 2 & 14,8 & 10,0 & 28,6 \\
\hline $\begin{array}{l}\text { Gobierno, sector privado, fundaciones } \\
\text { (extranjero) }\end{array}$ & 8 & 8 & 0 & 29,6 & 40,0 & 0,0 \\
\hline No aplica & 2 & 1 & 1 & 7,4 & 5,0 & 14,3 \\
\hline Contó con beca en la maestría & 132 & 100 & 32 & 100,0 & 100,0 & 100,0 \\
\hline No & 7 & 4 & 3 & 5,3 & 4,0 & 9,4 \\
\hline Conacyt & 99 & 77 & 22 & 75,0 & 77,0 & 68,8 \\
\hline $\begin{array}{l}\text { Gobierno, sector privado, fundaciones } \\
\text { (mexicanas) }\end{array}$ & 6 & 2 & 4 & 4,5 & 2,0 & 12,5 \\
\hline $\begin{array}{l}\text { Gobierno, sector privado, fundaciones } \\
\text { (extranjero) }\end{array}$ & 17 & 16 & 1 & 12,9 & 16,0 & 3,1 \\
\hline Organismos internacionales & 2 & 1 & 1 & 1,5 & 1,0 & 3,1 \\
\hline No aplica & 1 & 0 & 1 & 0,8 & 0,0 & 3,1 \\
\hline
\end{tabular}




\begin{tabular}{lrrrrrr}
\hline Cuadro 3. Trayectorias académicas y el rol central del Conacyt en la formación educativa de los miembros del \\
Sistema Nacional de Investigadores en el Exterior
\end{tabular}

Fuente: Encuesta para talentos mexicanos residentes en el exterior, Conacyt-UNESCO MOST México, 2014.

Un patrón visible y que vale la pena subrayar es que tanto en estudios de especialidad, maestría y doctorado, las mujeres prefirieron realizarlo en México que en el exterior, contrario al comportamiento de los varones, cuyo patrón es hacia un mayor porcentaje de ellos de contar con estudios en el exterior en mayores porcentajes. Este comportamiento se revierte cuando se analiza la estancia posdoctoral, donde ellas eligen en mayores porcentajes realizarlo en el exterior.

Un punto interesante de resaltar es que mientras que la migración por razones de estudio se da en el sentido sur-norte, la migración sur-sur es apenas perceptible. es a través de las figuras de estancia posdoctoral donde países del sur global aparecen como destinos con apenas $2,7 \%$.

El rol del Conacyt como financiador de estudios de posgrados es contundente, ya que de los que cuentan con estudios de especialidad, 22,2\% indicó que tuvo una beca Conacyt. En maestría este porcentaje llega a ser $75 \%$ y del $75,2 \%$ en doctorado. En cuanto a la estancia posdoctoral, es menos importante su participación $(26,4 \%)$ y aparecen gobiernos, fundaciones y sector privado extranjeros como financiadores de la estancia $(35,1 \%)$. Esto tiene sentido dado que el recién egresado de doctorado que ha estudiado en el extranjero cuenta ya con mayor información sobre este tipo de oportunidades en el país de destino.

\section{Trayectorias migratorias y académicas según razones de estudio y laborales}

En esta sección se analizan las trayectorias migratorias educativas y laborales. La manera en que se separaron, identificaron y analizaron las respuestas a la encuesta en combinación con los currículos, es que cuando la migración es motivada primordialmente por razones de estudio, de manera indirecta se puede desencadenar un segundo destino migratorio o la permanencia en el primer destino, sin que existan motivaciones claras para retornar. Esto se conjuga con la existencia de mayores conexiones, redes, vínculos en el destino, y no en el origen, que pueden propiciar la inserción laboral, sea en la universidad, centros de investigación o sector productivo. Mientras que cuando la migración es motivada por razones laborales, responde directamente a la necesidad de encontrar un mejor puesto laboral, mayor estabilidad en el contrato o mayores posibilidades de ascenso y/o paga; $y$, subsecuentemente, se pueden o no 
desencadenar procesos educativos en el lugar de destino.

Los patrones generales en ambas subsecciones son que, sin distinción entre área de estudio y sexo, la edad de la primera migración por razones de estudio suele estar entre los 25 y 29 años. Mientras que aquellos que migraron una vez que han terminado sus estudios de licenciatura (L), maestría (M) y doctorado (D) en México, la edad de la primera migración es después de los 30 años y en general, se trata de estancias posdoctorales.

Para aquellos que han realizado sus estudios en México (L, M, D) también se aprecia que antes del primer movimiento migratorio hubo una experiencia previa que se identifica como primera movilidad, y no es otra cosa que una movilidad o estancia corta, de 1 a 6 meses.

\subsection{Trayectorias por razones de estudio}

Para examinar estas trayectorias se tomaron en consideración aquellos casos que respondieron como razón de su emigración los estudios y que además adjuntaron su curriculum vitae. Adicionalmente, se buscó seleccionar representatividad según el área del conocimiento, el país de estudios del doctorado y contar con un balance de sexo; finalmente se tomaron todos los casos de mujeres y algunos de varones, dado que de ellos existía un mayor número de casos. Se trata entonces de 7 mujeres y 17 varones (cuadro 4 y diagrama 1).

\subsubsection{Física, matemáticas y ciencias de la tierra}

Las trayectorias educativas en cada uno de los casos, tanto para varones como para mujeres, han sido continuas; esto es, no han ocupado puestos laborales que no sean dentro de la academia; y en la mayoría de las ocasiones estos se realizan a la par de los estudios de posgrado impartiendo clases o en asistencia de investigación. No obstante, se aprecia que únicamente uno de los cinco ha logrado un puesto de trabajo más estable; el resto continúa realizando estancias posdoctorales.
Aunque para todos estos investigadores/as los estudios es la razón de la emigración, resalta el caso de Concepción de 55 años de edad, quien lleva 18 años residiendo en Canadá, pues ha logrado consolidar su vida profesional en ese país. Emigró para realizar sus estudios de doctorado y en el momento de la encuesta en 2014 ya había tenido puestos como profesora asistente y profesora asociada, previo a ser profesora en la Memorial University. Lo que se aprecia de su trayectoria laboral, al término de sus estudios doctorales, es un puesto como profesora de estadística e instructora por temporadas en esta misma universidad, Dalhousie. Posteriormente, realizó un posdoctorado y a continuación colaboró con la Universidad McGill como investigadora asociada.

Un caso interesante de retorno se observa con Jonathan $\mathrm{M}$, quien ha obtenido un puesto como investigador en las Cátedras Conacyt en 2014. Emigró a Alemania a los 21 años para realizar estudios de maestría y doctorado. En su trayectoria laboral durante sus estudios de doctorado, de manera paralela se desempeñaba como interno y asistente de investigación en la Universidad de Bremen. Al término de estos estudios, se desempeñó durante 10 años, de 2004 a 2013, como investigador en el Center for Industrial Mathematics en Alemania.

En estos y todos los demás casos consultados, se destaca que los y las investigadores/as expresaron alta correspondencia entre los estudios que realizaron y el puesto que ocupan, de igual modo indicaron un alto grado de satisfacción con el puesto y ocupación actual, excepto por Andrea, que indica un grado medio. La categoría no es distinta de la de sus pares y además cuenta en su historial innovativo con una patente. Su trayectoria laboral después de terminar el doctorado en 2009 ha continuado por dos estancias posdoctorales, una de tres años, 2009-2012, en la Johannes Kepler University, donde obtuvo el grado doctoral y otra que inició en 2012 y a la fecha de encuesta llevaba dos años, en el Leibniz Institute for Analytical Sciences. Pone de manifiesto la pregunta de ¿cuántos posdoctorados y de qué duración son pertinentes para obtener un puesto laboral más estable? 


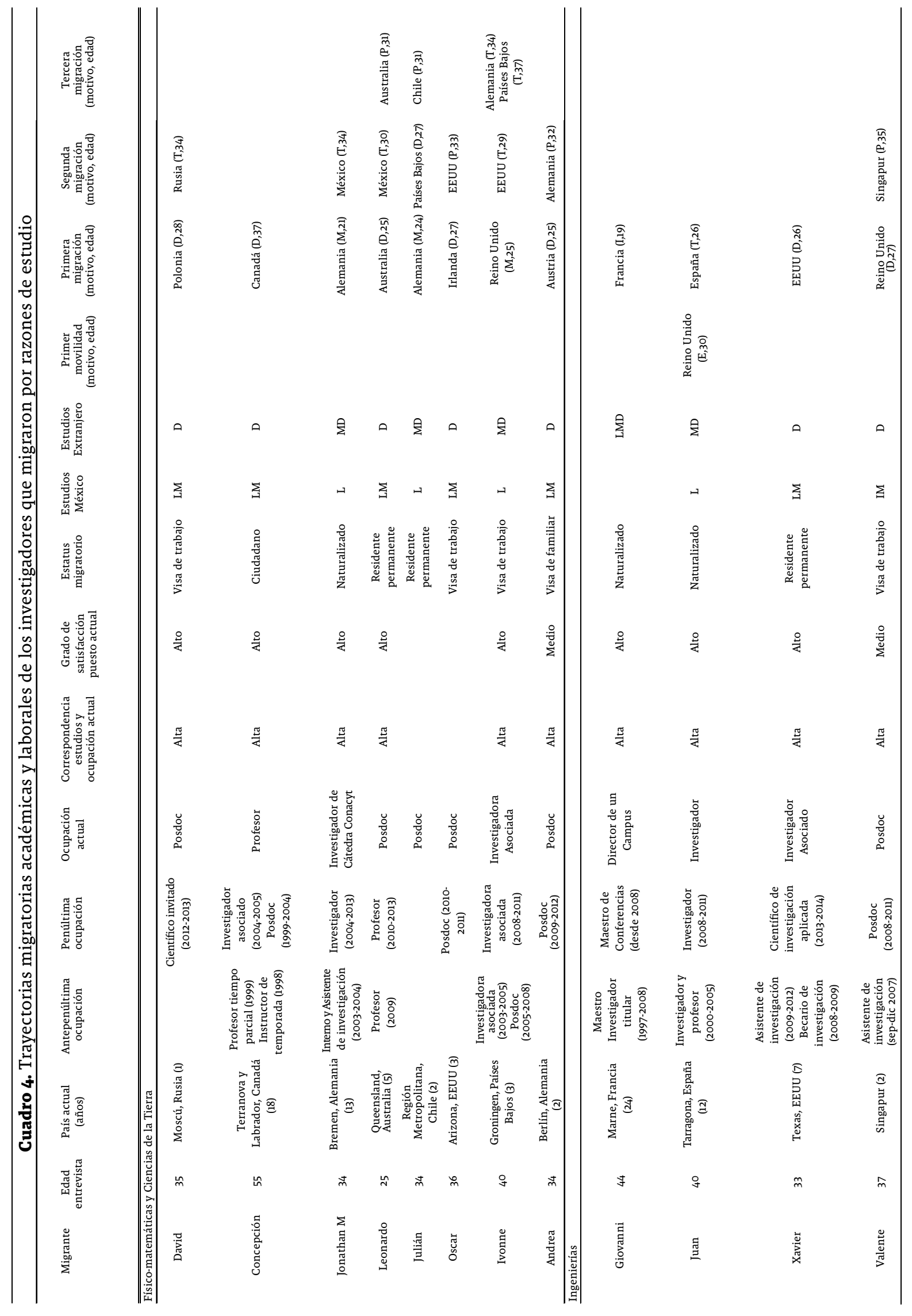




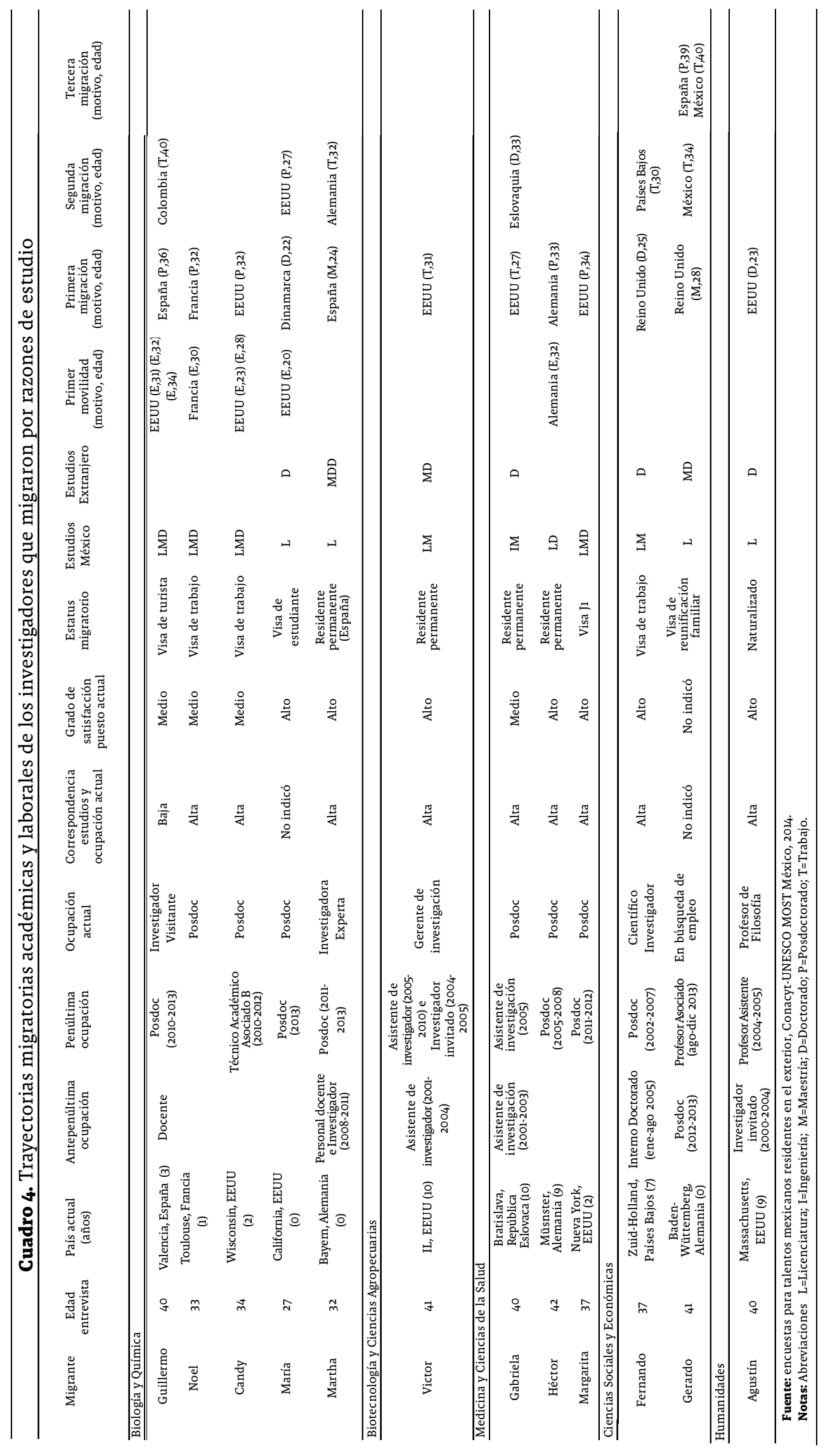




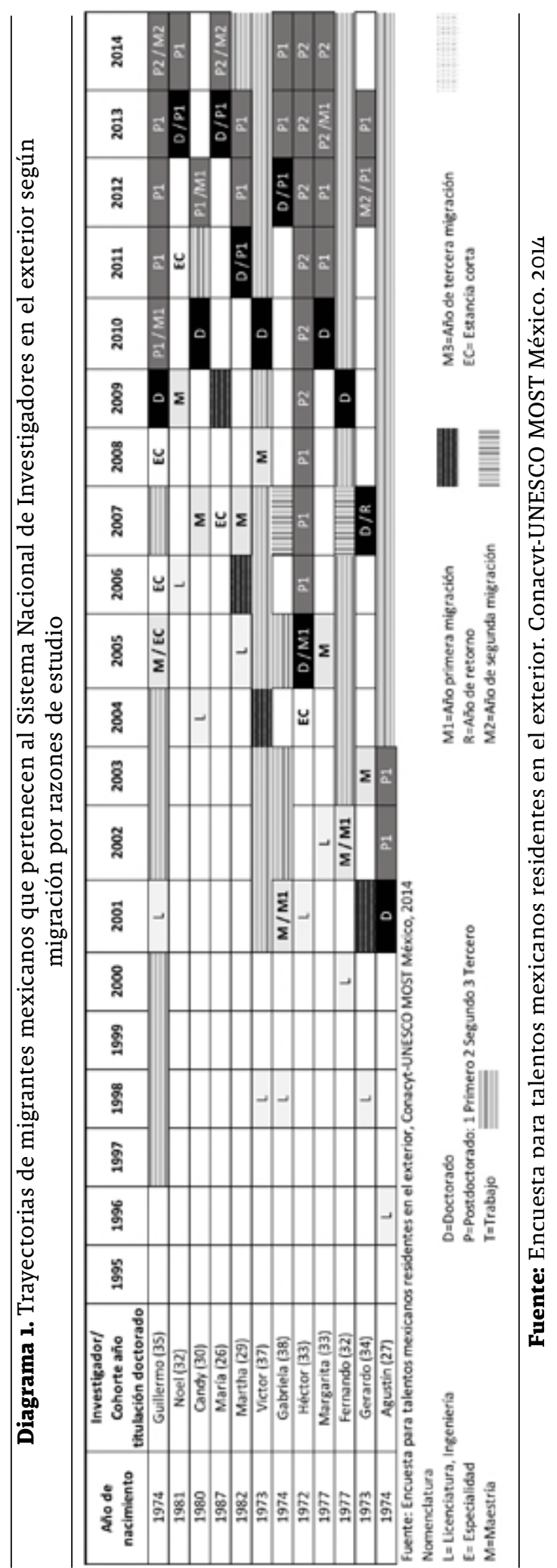

\subsubsection{Ingenierías}

Tres de las cuatro trayectorias laborales de estos investigadores/as muestran que obtienen puestos laborales estables en edades cercanas a los 40 años. Sobre estos investigadores/as, se revisan dos trayectorias.

La primera, de Giovanni, quien emigró a Francia a la edad de 19 años y básicamente realizó toda su formación en aquel país. Cuenta con una trayectoria laboral inmediata al término de sus estudios doctorales. Durante doce años, de 1997 a 2008, se desempeñó como maestro investigador titular del Instituto Nacional Politécnico de Lorraine. A continuación, funge como maestro de conferencias en el Arts et Métiers Paris Tech. Consolida su carrera a partir de 2010 en la École Nationale Supériure d'Arts et Métiers.

En el caso de la trayectoria de Valente, de 37 años de edad, realizó estudios de doctorado en Reino Unido y a la par trabajó como asistente de investigación en la misma universidad. Al término de sus estudios, realizó una estancia posdoctoral en la Universidad de Nottingham, de 2008-2011. En 2012 inició una nueva estancia posdoctoral en la National University of Singapore. Un indicio de que busca un puesto con mayor estabilidad es el grado de satisfacción medio que dijo tener en cuanto al puesto y ocupación que desempeña. No obstante, manifiesta una alta correspondencia entre su formación académica y la ocupación que tiene.

\subsubsection{Biología y química}

Tres de los cinco investigadores, de hecho, los varones, realizaron toda su formación educativa en México, mientras que las investigadoras únicamente hicieron sus estudios de licenciatura. De estos cinco investigadores, tres de ellos realizan una estancia posdoctoral y únicamente dos de ellos, Martha y Guillermo han logrado tener un puesto más estable.

En el caso de Martha con apenas 32 años, muestra una trayectoria académica y laboral bastante prominente y continua. Ha vivido en España durante 8 años y cuenta con residencia 
permanente. En 2006 inició sus estudios de maestría, y al término de estos realizó estudios doctorales en dos universidades, terminándolos en 2011. Mientras estudiaba, de manera paralela trabajó como personal docente e investigador de 2008 a 2011 en una de las universidades donde obtuvo el grado doctoral. Inmediatamente después hizo una estancia posdoctoral, de 20112013, en el Institut Català d'Investigació. Y desde inicios de 2014 funge como investigadora experta (Acciones Marie Curie de la Unión Europea) y Polymaterials AG. Derivado de su trabajo, se encuentra en Alemania. Indica un alto grado de satisfacción con el puesto y ocupación que desempeña y una alta correspondencia entre sus estudios y la ocupación que realiza.

En el caso de Candy, de 34 años, cursó todos sus estudios en México. En 2010 se graduó del doctorado en San Luis Potosí, donde a continuación se desempeñó por dos años como técnica académica asociada B. En 2012 inició una estancia posdoctoral en la Universidad de Wisconsin-Madison. Aunque señala que existe una alta correspondencia entre su ocupación y los estudios que ha realizado, menciona un grado de satisfacción medio en cuanto al puesto que tiene.

Por su parte, tanto Noel como Guillermo señalan un nivel medio de satisfacción en este sentido.

\subsubsection{Biotecnología y ciencias agropecuarias}

Víctor, de 41 años de edad, emigró a Estados Unidos por razones de estudio, donde realizó maestría y doctorado, pero regresó a México en el 2001, y estuvo hasta el 2004, cuando regresó a estados Unidos, donde ya es residente permanente. Señala que de 2004 a 2005 funge como profesor invitado en la Universidad de Illinois donde previamente había realizado sus estudios de posgrado; posteriormente, por cinco años y hasta 2010, laboró en esta misma universidad como asistente de investigador. Finalmente, desde 2010 se desempeña como gerente de Investigación en Nutrición de No Rumiantes.
Señala la categoría salarial más alta, 60 mil dólares anuales y más. Asimismo, señala un alto grado de satisfacción entre el puesto y ocupación que tiene y una alta correspondencia entre lo que estudió y su ocupación.

\subsubsection{Medicina y ciencias de la salud}

Tres investigadores, Gabriela de 40 años, Héctor de 42 y Margarita 37, al momento de la encuesta se encontraban realizando una estancia posdoctoral. Únicamente Gabriela realizó estudios de posgrado en el exterior y es también la que indica un nivel medio de satisfacción con respecto al puesto y ocupación que tiene, a diferencia de Héctor y Margarita quienes indicaron un nivel alto.

Más en detalle, se observa que Gabriela lleva 10 años en la República Checa, donde ya es residente permanente. En el 2012 terminó sus estudios de doctorado en Eslovaquia, no obstante, su trayectoria educativa no fue continua. Al término de sus estudios de maestría en México se colocó como asistente de investigación en Estados Unidos; en 2005 regresó a México y trabajó como asistente de investigación en la UNAM. En 2007 realiza una tercera migración hacia Eslovaquia por cuestiones de estudio. Finalmente, en 2011 inicia una estancia posdoctoral en la Academia de Ciencias en Eslovaquia.

Héctor, por su parte, tiene 42 años y 9 de vivir en Alemania, país donde ya tiene el estatus migratorio de residente permanente. Sus estudios los realizó en México y al finalizar el doctorado emigró a Alemania para realizar una estancia posdoctoral durante tres años en la Universidad de Heidelberg. Al finalizar esta estancia en 2008, inició otra en la Universidad de Münster y en el momento de la encuesta aún se encontraba en ella.

\subsubsection{Ciencias sociales y económicas}

Dos investigadores, Fernando y Gerardo, tienen condiciones de actividad opuestas. Fernando con 37 años ya tiene un cierto grado de consolidación en el trabajo; mientras que Gerardo de 41 años se encuentra en búsqueda de empleo. 
La diferencia en términos del estatus migratorio es que mientras que Fernando tiene una visa de trabajo para un migrante altamente cualificado, Gerardo tiene una visa de reunificación familiar toda vez que su esposa es alemana.

Fernando estudió su licenciatura y maestría en México; en 2002 emigró a Reino Unido para estudiar su doctorado. A la par de sus estudios realizó una práctica laboral en el UNU-MERIT en 2005, y de 2002 a 2007 fungió como investigador de posgrado. De 2007 a la fecha, se desempeña como investigador científico en un centro de investigación europeo prestigioso.

Gerardo hizo su licenciatura en México y la maestría y doctorado de manera continua en Reino Unido. La migración por razones de estudio ocurrió en 2001 y en 2007, al obtener el último grado, fue beneficiario de una beca de retorno por parte del Conacyt. En 2012, se instaló en Deusto para realizar una estancia posdoctoral. Y en 2013, de agosto a diciembre, colaboró como profesor asociado en México. Por último, en 2014, al momento de la encuesta se encontraba en Alemania en búsqueda de empleo.

\subsubsection{Humanidades}

En el caso de Agustín, de 40 años de edad, lleva nueve viviendo en Estados Unidos. Sus estudios de licenciatura los realizó en México y la migración por razones de estudio la llevó a cabo en 1997; al finalizarlos, se incorporó como investigador en la Universidad de St Andrews. Enseguida, de 2004 a 2005, trabajó en la Universidad de California como asistente de profesor. Finalmente, en el MIT inició en 2005 como profesor asociado, logrando la titularidad en 2008. Desde 2014 es profesor de Filosofía.

\subsection{Trayectorias por razones laborales}

$\mathrm{Al}$ igual que en la subsección previa, se parte de que la lógica del proceso migratorio por razones laborales está directamente ligada a una necesidad de encontrar un mejor puesto laboral, mayor estabilidad en el contrato, mayores posibilidades de ascenso y/o mayor paga. Incluso en esta lógica puede introducirse la idea de ascenso o movilidad social a través del nivel educativo, en países donde existen condiciones estructurales que lo permiten.

Se seleccionaron aquellos individuos que respondieron simultáneamente como razón de emigración: a) mayores ingresos, b) mejores condiciones y c) oportunidades laborales. Se tomó en consideración un balance tomando en consideración el sexo, el país donde estudió su doctorado y el área del conocimiento. Se trata de 6 mujeres y 8 varones (cuadro 5 y diagrama 2).

\subsubsection{Física, matemáticas y ciencias de la tierra}

En esta área sobresalen dos casos particulares: en el primero, solo uno de los cinco investigadores tiene un puesto laboral distinto a una estancia posdoctoral; en el segundo caso, tres de los cinco investigadores realizaron los estudios de licenciatura, maestría y doctorado $(L, M, D)$ en México.

El primer caso, la trayectoria académica de Eva muestra que antes del primer evento migratorio para estudiar el doctorado realizó una estancia corta en Canadá a la edad de 22 años. A los 25 años inició sus estudios doctorales en Estados Unidos, para luego realizar una estancia posdoctoral en Alemania a la edad de 31 años y, por último, emigra a Estados Unidos, donde a la fecha de la encuesta se desempeñaba como investigadora científica. En este puesto, a pesar de que expresó que la correspondencia entre los estudios y su ocupación es alta, el grado de satisfacción con su puesto es medio.

En el segundo caso, Bernardo, después de concluir sus estudios doctorales hizo una estancia posdoctoral en la República de Corea a la edad de 28 años. Al término de esta fue a China, donde hizo un segundo posdoctorado. $\mathrm{Y}$ a la edad de 32 años, ha vuelto a la República de Corea y se encontraba realizando su tercera estancia posdoctoral al momento de la entrevista. 


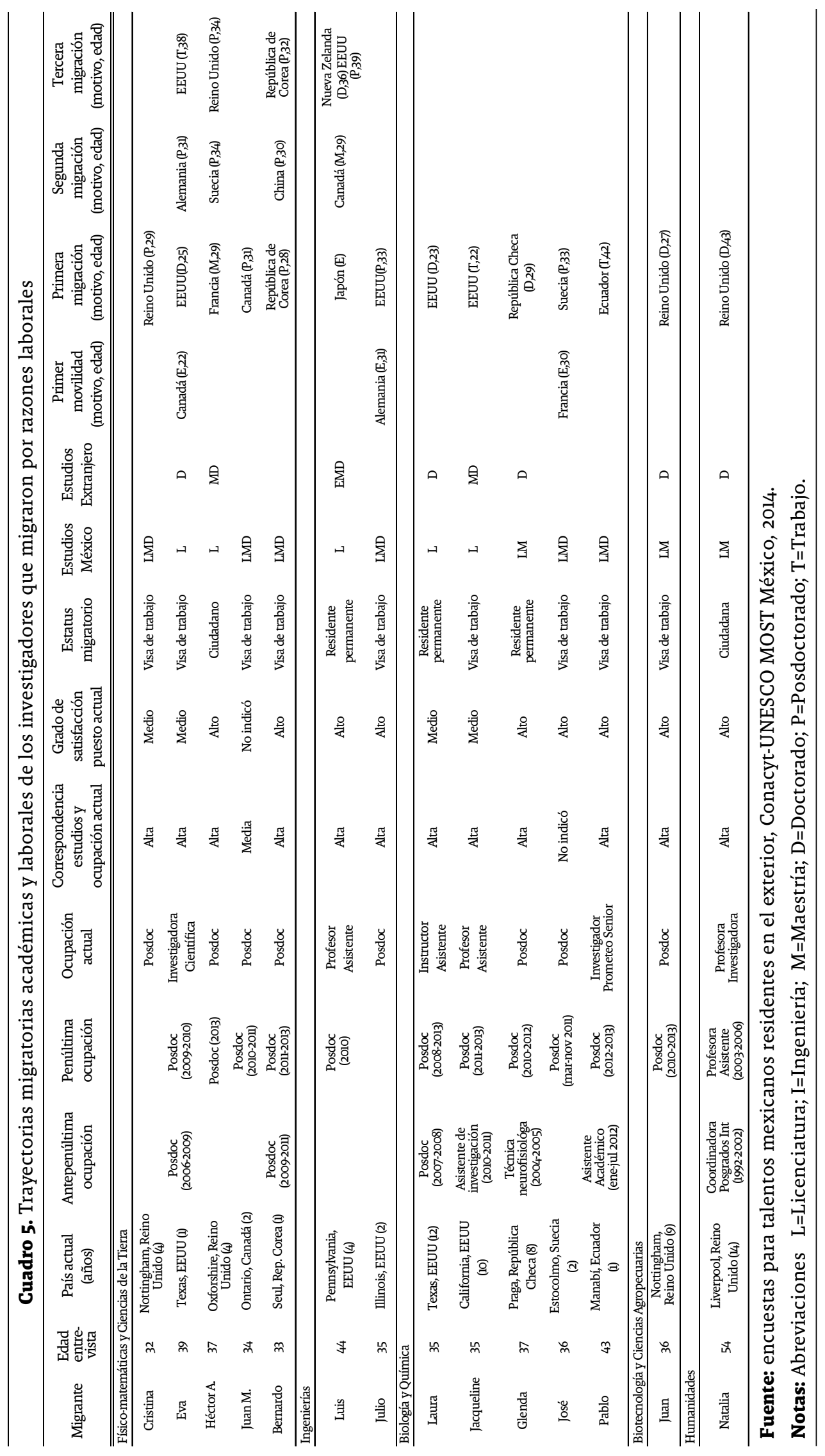




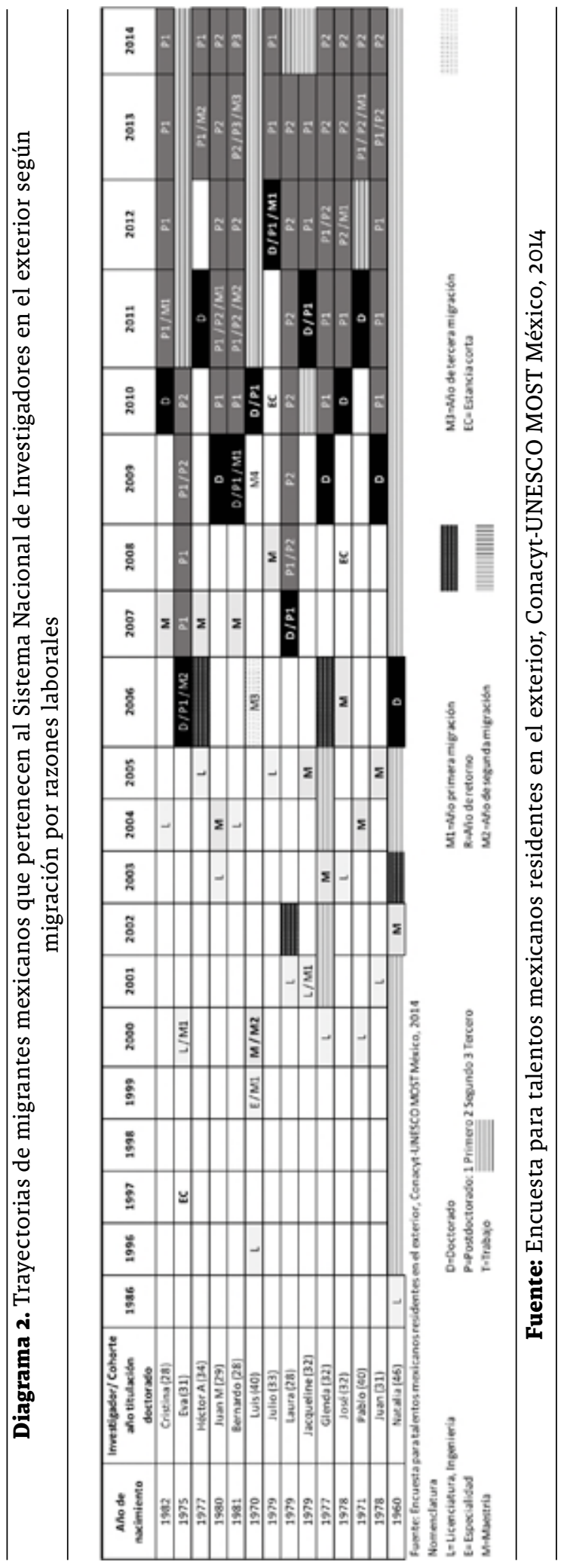

\subsubsection{Ingenierías}

Se presentan dos casos: Luis, quien realizó únicamente la licenciatura en México y los estudios de especialidad (E), maestría (M) y doctorado(D) en el extranjero; $y$, Julio, quien estudió la licenciatura $(\mathrm{L})$, la maestría (M) y el doctorado (D) en México. En el primer caso, se aprecian varias experiencias migratorias relacionadas con los estudios: Japón, Canadá y Nueva Zelanda; el último movimiento hacia Estados Unidos se relaciona con una estancia posdoctoral. De hecho, cuenta con residencia permanente en este país y afirmó una alta correspondencia entre los estudios que realizó y la ocupación que desempeña, así como un alto grado de satisfacción en su puesto actual.

En el segundo caso, Julio, de igual manera indica una alta correspondencia entre los estudios que realizó y la ocupación que desempeña, así como un alto grado de satisfacción en su puesto actual. En cuando a su experiencia migratoria, previo a la estancia posdoctoral que realizó a los 33 años, hizo una estancia corta de menos de 6 meses. Lo anterior indica que puede existir una mayor propensión en la movilidad internacional en recién egresados que se vinculan con investigadores y/o instituciones extranjeras mientras realizan estudios.

\subsubsection{Biología y química}

En este grupo de investigadores, se aprecian nuevamente dos patrones: el primero, tres de cinco realizaron maestría y/o doctorado en el extranjero; el segundo, tres de los cinco se encuentran realizando estancias posdoctorales (Pablo, aunque está como Prometeo Senior ${ }^{5}$, se trata de estancia posdoctoral y no de un puesto permanente).

Como ejemplo del primer caso, Jacqueline de 35 años de edad lleva 10 años viviendo en Estados Unidos y cuenta con visa de trabajo. Su primera

5 El proyecto Prometo es una iniciativa del gobierno ecuatoriano para fortalecer la investigación en el país a través de la docencia, la transferencia de conocimientos; vinculando a investigadores extranjeros y ecuatorianos residentes en el exterior. Las estancias Prometeos van de 6 meses a 1 año. 
migración fue a los 22 años por razones de trabajo, no obstante, más tarde realizó estudios de maestría y doctorado en este país. Su trayectoria laboral muestra que se ha desempeñado en el ámbito de la academia; realizó una estancia posdoctoral y actualmente funge como profesora asistente. En su caso, advierte que es alta la correspondencia entre su formación y su ocupación actual, pero es medio el grado de satisfacción con el puesto actual que desempeña.

En el caso de José de 33 años, su razón migratoria fue realizar una estancia posdoctoral en Francia, al término de la cual se dirigió a Suecia, donde se encuentra haciendo una segunda estancia posdoctoral. De hecho, José cursó sus estudios de licenciatura, maestría y doctorado en México. En la encuesta expresó contar con un alto grado de satisfacción con el puesto actual que desempeña.

\subsubsection{Biotecnología y ciencias agropecuarias}

En el caso de Juan, se aprecia que su primera experiencia migratoria fue a la edad de 27 años y estuvo relacionada con los estudios doctorales. Al término de estos, realizó una estancia posdoctoral y actualmente se encuentra en su segunda estancia. Concomitante a ello, se aprecia que cuenta con permiso de trabajo y que tanto la correspondencia entre lo que estudió y la ocupación que desempeña, como el grado de satisfacción con su puesto actual son altos.

\subsubsection{Humanidades}

En esta área solo se presenta la experiencia de Natalia, quien a pesar de haber respondido que sus motivaciones migratorias eran de índole laboral, su trayectoria muestra que la primera experiencia está vinculada a las razones de estudios doctorales. De hecho, es un caso atípico en términos de la edad en que inició con el doctorado (43 años), cuando en general, los datos presentados muestran edades menores a 40 años. No obstante, su perfil laboral se muestra consolidado dado que ha vivido en Reino Unido durante 14 años, cuenta con ciudadanía y se desempeña en un puesto de profesora investigadora.

\section{Conclusiones}

De manera más general, los patrones que se pudieron identificar fueron: 1) la migración por razones de estudio se realiza en edades inferiores a los 30 años y las estancias posdoctorales pasando esta edad. 2) La exposición al sistema educativo e investigativo abre la posibilidad de realizar trayectorias académicas continuas en los países de destino. Esto configura la posibilidad de que se trate de una migración más permanente, mientras que, cuando la primera migración involucra una estancia posdoctoral, es más probable que la migración sea de carácter temporal.

En este trabajo se observa que las trayectorias migratorias se articulan a las razones laborales y de estudios conformando así un solo entramado. Las razones laborales y de estudio que definen la emigración de este grupo toman diversa importancia de acuerdo con otras categorías como son el sexo, el área de estudio y el país donde estudió. De esta primera conclusión surgen algunas preguntas de investigación futuras: ¿Indagar los procesos que llevan a una migración de tipo permanente a partir de la búsqueda de estudios en el extranjero? ¿Cuál es el papel que están jugando las redes personales y laborales que se construyen a la par de los procesos migratorios? ¿En qué medida se puede considerar la figura del posdoctorado como una estrategia para lograr una inserción laboral fija y evitar el retorno?

Una conclusión que es importante resaltar de este análisis es el retorno de este grupo de investigadores/as al país. Los resultados encontrados ponen en discusión, una vez más, la categoría "fuga de cerebros" y la repatriación de estos investigadores/as dado que discursivamente se les concibe como potencial perdido para generar e impulsar procesos de desarrollo nacionales. Se observó que a nivel personal se construyen redes y/o vínculos con el país, no obstante, los vínculos institucionales son débiles, lo que 
denota que la cuestión estructural sigue desempeñando un rol central para pensar el diseño e implementación de políticas que articulen el tema de la migración calificada a la estrategia de desarrollo del aparato productivo.

Una línea de investigación que surge de estos resultados analizados es el nivel de insatisfacción con el puesto que tienen las mujeres así como los bajos niveles salariales. Dadas las limitaciones de la información analizada, no se pueden formular conclusiones pero se puede visualizar la necesidad de responder a preguntas como: ¿Por qué las investigadoras están menos satisfechas con sus puestos de trabajo aunque la correspondencia entre las actividades que realizan y su formación académica es alta? Para tratar de responder a esta pregunta puede ser útil la perspectiva de la interseccionalidad pero enfatizando en las categorías de género y clase y no únicamente en las categorías de sexo, área del conocimiento y país de estudios doctorales, como se hizo en este trabajo.

\section{Referencias}

Anthias, F. (2006). Género, etnicidad, clase y migración: interseccionalidad y pertenencia translocalizacional. En P. Rodríguez (Ed.), Feminismos periféricos (pp. 49-68). Granada, España: Editorial Alhulia.

Calva-Sánchez, L. E. (2014). Migración calificada de mexicanos a Estados Unidos: entre la migración tradicional y el desarrollo profesional. Recuperado de http://xiireuniondemografica.ibero.mx/pdf/ resumen_extenso/6.6.1.pdf

Calva-Sánchez, L. E. y Carrión, V. (2014). Una mirada crítica a la migración calificada desde México a Estados Unidos. Coyuntura Demográfica, 5, 39-63.

Chávez, M. G. y Gaspar, S. (2018). Análisis salarial de las mujeres inmigrantes calificadas en Estados Unidos. En R. Soto y A. Girón (Ed.), Repensando la migración desde un enfoque de género: proyectos productivos y financiamiento (en prensa). Ciudad de México, México: UNAM-ONU Mujeres-UAZ.

Delgado-Wise, R. y Chávez, M. G. (2015). Claves de la exportación de fuerza de trabajo calificada en el capitalismo contemporáneo: lecciones de la experiencia Mexicana. Migración y Desarrollo, 13(25), 3-32.

Delgado-Wise, R. y Chávez, M. G. (2016). Migración calificada: entre la pérdida de talento y la oportunidad de transformar a México con innovación. Revista Mexicana de Política Exterior, (107), $117-135$.

Delgado-Wise, R., Chávez, M. G. y Ramírez, H. (2016). La innovación y la migración calificada en la encrucijada: Reflexiones a partir de la experiencia Mexicana. REHMU- Rev. Intersdiscip. Mobil. Hum., XXIV(47), 153-174.

Elder, G. (1985). Life Course Dynamics. Trajectories and Transitions. Ithaca, US: Cornell University Press.

Ezquerra, S. (2008). Hacia un análisis interseccional de la regulación de las migraciones: la convergencia de género, raza y clase social. En E. Santamaría (Ed.), Retos epistemológicos de las migraciones transnacionales (pp. 237-260). Barcelona, España: Anthropos. 
Gaspar, S. (2015). ¿Estudiar para emigrar o emigrar para estudiar? Proceso de integración de los inmigrantes mexicanos calificados en Estados Unidos (tesis de maestría). Universidad Nacional Autónoma de México, Ciudad de México, México.

Gaspar, S. y Chávez, M. (2016). Migración mexicana altamente calificada: 1990-2013. Problemas del Desarrollo, 47(185), 81-110.

Lozano-Ascencio, F. y Gandini, L. (2012). La migración calificada de México a Estados Unidos: tendencias de la década 2000-2010. Coyuntura demográfica, 2, 51-55.

Lozano-Ascencio, F., Gandini, L. y Ramírez-García, T. (2015). Devaluación del trabajo de posgraduados en México y migración internacional: los profesionistas en ciencia y tecnología. Migración y Desarrollo, 13(25), 61-89.

Ramírez-García, T. y Gandini, L. (2017). Trabajadoras calificadas: las mujeres mexicanas en el Mercado de trabajo estadounidense en perspectiva comparada. Revista Latinoamericana de Población, 10(19), 33-56.

Tuirán, R. y Ávila, J. L. (2013). Migración calificada entre México-Estados Unidos. Desafíos y opciones de política pública. Migración y Desarrollo, 11(21), 43-63. 
Mónica Chávez Elorza • Yolanda Alfaro 